\title{
Sunspot group tilt angles and the strength of the solar cycle (Corrigendum)
}

\author{
M. Dasi-Espuig ${ }^{1}$, S. K. Solanki ${ }^{1,2}$, N. A. Krivova ${ }^{1}$, R. Cameron ${ }^{1}$, and T. Peñuela ${ }^{1,3}$ \\ 1 Max-Planck-Institut für Sonnensystemforschung, Max-Planck-Str. 2, 37191 Katlenburg-Lindau, Germany \\ e-mail: dasi@mps.mpg.de \\ 2 School of Space Research, Kyung Hee University, Yongin, 446-701 Gyeonggi, Korea \\ 3 Astronomical Institute, St. Petersburg State University, Russia
}

A\&A 518, A7 (2010), DOI: 10.1051/0004-6361/201014301

Key words. sunspots - Sun: dynamo - magnetic fields - Sun: activity - errata, addenda

In the published version of Dasi-Espuig et al. (2010), Fig. 4a shows the relationship between the cycle averaged tilt angle normalised by the mean latitude, $\langle\alpha\rangle /\langle\lambda\rangle$, based on the Mount Wilson (MW) data, and the strength, S, of the same cycle. This figure is incorrect since the values of the cycle average tilt angles used, $\langle\alpha\rangle$, were based on the Kodaikanal (KK) data, while the cycle average latitudes, $\langle\lambda\rangle$, were based on the MW data. This error also affected the correlation coefficients between the cycle area-weighted tilt angles normalised by the mean latitude, $\left\langle\alpha_{\omega}\right\rangle /\langle\lambda\rangle$, and the $\mathrm{S}$ of the same cycle.

Here we present the correct plot in Fig. 1 using the MW data for both the cycle average tilt angles and latitudes. Figure 1 is now in agreement with the results of Ivanov (2012), and of McClintock \& Norton (2013) who also employed the MW data in a similar analysis. The corresponding correlation coefficients are listed in Table 1 together with the probability that these are due to chance. Also listed are the correlation coefficients of the relationships using $\left\langle\alpha_{\omega}\right\rangle /\langle\lambda\rangle$.

We want to stress that this error only affected the relationships of the normalised cycle average and cycle area-weighted average tilt angles based on the MW data, with the S of the same cycle. Therefore, all of the results based on the KK data, and the results based on the MW data of the relationships with the $\mathrm{S}$ of the next cycle remain unchanged. Since the correlation coefficient of the corrected anticorrelation between $\langle\alpha\rangle /\langle\lambda\rangle$ and the $\mathrm{S}$ of the same cycle is weaker than in the published version, point (3) in Sect. 5 should say "A negative correlation between the normalised tilt angle, or $\langle\alpha\rangle /\langle\lambda\rangle$, and the strength of the same cycle is found $\left(r_{\mathrm{c}}=-0.79\right.$ and $r_{\mathrm{c}}=-0.93$ for MW and KK, respectively)". The rest of the conclusions remain unchanged.

\section{References}

Dasi-Espuig, M., Solanki, S. K., Krivova, N. A., Cameron, R., \& Peñuela, T. 2010, A\&A, 518, A7

Ivanov, V. G. 2012, Geomagnetism and Aeronomy, 52, 999

McClintock, B. H., \& Norton, A. A. 2013, Sol. Phys., in press [arXiv: 1305.3205]

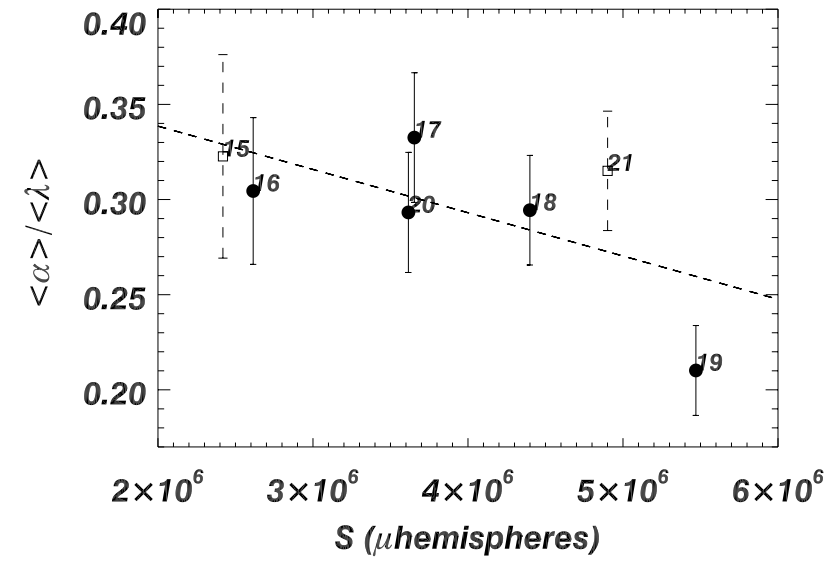

Fig. 1. Corrected Fig. 4a of Dasi-Espuig et al. (2010). Cycle averaged tilt angle normalised by the mean latitude vs. the strength of the same cycle based on MW data $\left(r_{\mathrm{c}}=-0.79\right)$. The error bars represent $1 \sigma$ error and the dashed line a linear fit to the points. Cycles 15 and 21 are shown as squares with dashed lines for the error bars because of their incompleteness.

Table 1. Corrected correlation coefficients shown in Table 2 of DasiEspuig et al. (2010), $r_{\mathrm{c}}$, between the four quantities described in the published version based on the tilt angle and the strength (S), amplitude (A), and length (L) of the same cycle, and the probability that the correlation is due to chance, P, for the MW (cycles 16-20) data set.

\begin{tabular}{lccccccc}
\hline \hline & \multicolumn{7}{c}{ Mount Wilson } \\
\hline Parameter & \multicolumn{2}{c}{$\mathrm{S}$} & \multicolumn{2}{c}{$\mathrm{A}$} & \multicolumn{2}{c}{$\mathrm{L}$} \\
\hline & $r_{\mathrm{c}}$ & $\mathrm{P}$ & $r_{\mathrm{c}}$ & $\mathrm{P}$ & $r_{\mathrm{c}}$ & $\mathrm{P}$ \\
\hline$\langle\alpha\rangle /\langle\lambda\rangle$ & -0.79 & 0.11 & -0.80 & 0.10 & -0.12 & 0.85 \\
$\left\langle\alpha_{\omega}\right\rangle /\langle\lambda\rangle$ & -0.67 & 0.22 & -0.68 & 0.21 & -0.32 & 0.60 \\
\hline
\end{tabular}

